$(\mathrm{MAF}<5 \%)$ SNPs at one locus in gene-centric analysis of CAD. We hypothesised that patients with $\mathrm{CAD}$ will show over-representation of rare alleles compared to controls.

Methods To examine associations between rare alleles and CAD, we have used data from $2119 \mathrm{CAD}$ cases and 2440 healthy controls recruited to the Welcome Trust Case-Control Consortium (WTCCC) Study. DNA from each subject was genotyped for approximately 45000 SNPs in more than 2000 genes/loci using 50K IBC array (version 1). Association analysis was based on the CCRaVAT (Case-Control Rare Variant Analysis Tool) algorithm that maximises statistical power by combining all rare alleles within defined regions into a single "super locus". Differences in the proportion of cases and controls carrying rare "super loci" were tested by Pearson's or Fisher's exact test. Empirical p values were generated by permuting case-control status a predefined number of times and repeating the analysis for each replicate.

Results 5 candidate regions (MMP23B, VEGFA, DVL1, RIPK1, LPAL2) showed an over-accumulation of rare alleles in patients with $\mathrm{CAD}$ when compared to controls $(\mathrm{FDR}<50 \%)$. The number of analysed rare alleles at each of these loci ranged from 4 to 42 . The most significant over-representation of rare variants were identified at MMP23B (matrix metallopeptidase 23B gene; $\mathrm{p}=1.3 / 10^{4}$ ), a gene previously unsuspected to play a major role in CAD and VEGFA (vascular endothelial growth factor $A ; p=2.6 \times 10^{-4}$ ). Only one of the identified genes (LPAL2; $p=1.7 \times 10^{-3}$ ) lies within the locus that was previously shown to harbour rare variants associated with susceptibility to CAD

Conclusions Rare alleles are associated with predisposition to $\mathrm{CAD}$ and this gene-centric analysis combining information from lowfrequency variants of the same locus has a potential to uncover, at least a proportion of, the "missing heritability" of CAD.

\section{GENOME WIDE METHYLATION ANALYSIS IN CORONARY ARTERY DISEASE}

doi:10.1136/heartjnl-2011-300198.69

K J Dick, C P Nelson, P S Braund, A H Goodall, N J Samani. University of Leicester, Leicester, UK

Background Using genome-wide association studies several genes have been identified that affect the risk of CAD. However, these genes only explain part of the heritability. There is increasing evidence of the role of epigenetic regulation in complex diseases that may explain part of the missing heritability. DNA methylation is an important epigenetic change that regulates gene expression. Any role of methylation in $\mathrm{CAD}$ is poorly understood. Therefore we undertook an exploratory genome-wide screen to identify genes differentially methylated in CAD cases and controls.

Methods We characterised DNA methylation in 24 CAD patients with a documented history of MI and 24 matched controls from the Cardiogenics case-control cohort. All subjects were male, ranging in age from 40 to 57 years. For each subject, genomic DNA, isolated from whole blood, was bisulphite converted and run on Illumina HumanMethylation 27 bead chips. The HumanMethylation 27 chips interrogate $27578 \mathrm{CpG}$ sites spanning 14495 genes with an average of 2 CpG sites per gene.

Results Global DNA methylation level was significantly higher in cases compared to controls $\left(p=9.0 \times 10^{-4}\right)$. Furthermore, 686 individual CpG sites, spanning 633 genes showed statistically significant differences in methylation levels between cases and controls. Significant signals after Bonferroni correction for multiple comparisons included GNAS $\left(p=7.94 \times 10^{-5}\right)$, which is involved in receptormediated signal transduction, PCMT1 $\left(p=7.94 \times 10^{-5}\right)$, ACD $\left(p=3.48 \times 10^{-4}\right.$ part of the telosome/shelterin complex), ATXN2 and APOA1 $\left(p=5.6 \times 10^{-3}\right.$ and $\left.p=0.01\right)$. To explore the potential func- tional importance of differences in methylation level in cases and controls for individual genes, we examined the relationship of methylation level to transcript level in monocytes and macrophages on a gene by gene basis and identified several genes including GNAS and PCMT1 that showed significant correlations between gene expression and methylation. Pathway enrichment analysis of the differentially methylated genes using the DAVID bioinformatics resource identified a number of pathways that showed significant enrichment including the calcium signalling pathway $\left(p=3.85 \times 10^{-7}\right)$.

Conclusions This pilot study has shown several significant differences in gene methylation patterns between $\mathrm{CAD}$ cases and controls. We also found a correlation between methylation level and gene expression for a number of these genes. Genes differentially methylated in $\mathrm{CAD}$ are significantly enriched for a number of pathways including the calcium signalling pathway. While these findings require further validation they suggest that epigenetic changes may play an important role in the pathogenesis of CAD.

\section{GENE EXPRESSION AT THE 9p21 LOCUS AND CAD RISK}

doi:10.1136/heartjnl-2011-300198.70

${ }^{1} \mathrm{C}$ P Nelson, ${ }^{2} \mathrm{P}$ Lundmark, ${ }^{1} \mathrm{~V}$ Codd, ${ }^{1} \mathrm{~A}$ H Goodall, ${ }^{2} \mathrm{~A}$ C Syvänen, ${ }^{1} \mathrm{~N}$ J Samani. ${ }^{1}$ Department of Cardiovascular Sciences, University of Leicester, Glenfield Hospital, Leicester, UK; ${ }^{2}$ Department of Medical Sciences, Molecular Medicine, Science for Life Laboratory, Uppsala University, Uppsala, Sweden

Background Human chromosome 9p21 harbours a locus that affects risk of coronary artery disease (CAD) through an unknown mechanism. The variants at the locus most strongly associated with CAD lie in non-coding regions suggesting that the affect on $\mathrm{CAD}$ risk may be mediated through regulation of gene expression. We investigated the association of single nucleotide polymorphisms (SNPs) across the locus with expression of genes in the locus and compared this with association of the same SNPs with CAD risk.

Methods We quantified transcript levels for CDKN2A, CDKN2B, ARF and MTAP in circulating monocytes from 422 healthy blood donors and $386 \mathrm{CAD}$ cases and obtained genotypes for SNPs in the 9 p21 region in the same subjects using genome-wide platforms. We also quantified allelic expression (AE) for these genes and for ANRIL in 186 of the healthy blood donors. We compared expression quantitative trait loci (eOTL) associations for the genes with association findings for the same SNPs for CAD in the Wellcome Trust Case Control Consortium study.

Results In the global gene expression analysis, we found strong cis eQTLs for both CDKN2B $\left(\mathrm{p}=1.3 \times 10^{-38}\right)$ and MTAP $\left(p=6.6 \times 10^{-23}\right)$, explaining $17.0 \%$ and $8.0 \%$ of the expression of these genes. AE analysis confirmed these findings (CDKN2B, $p=6.0 \times 10^{-64}$; MTAP, $\left.p=1.4 \times 10^{-38}\right)$ and also showed a significant cis-eQTL effect on ANRIL expression $\left(p=3.5 \times 10^{-28}\right)$. Interestingly, the SNPs associated with CDKN2B and ANRIL expression were the same. However, the SNPs showing e-OTL effects were distinct from SNPs that showed an association with CAD risk $\left(p=2.2 \times 10^{-12}\right)$. Even in the region with a physical overlap of variants affecting expression of CDKN2B/ANRIL and CAD risk, the effects of the respective variants were independent of each other. Expression of CDKN2A and ARF was low but did not show any obvious eQTL effect, or differences according to genotype at CAD-associated SNPs.

Conclusions Our findings in monocytes do not support the hypothesis that the chromosome 9p21 locus mediates CAD risk by affecting expression of the genes at the locus. The mechanism by which the chromosome 9p21 locus affects CAD risk requires further elucidation. 